
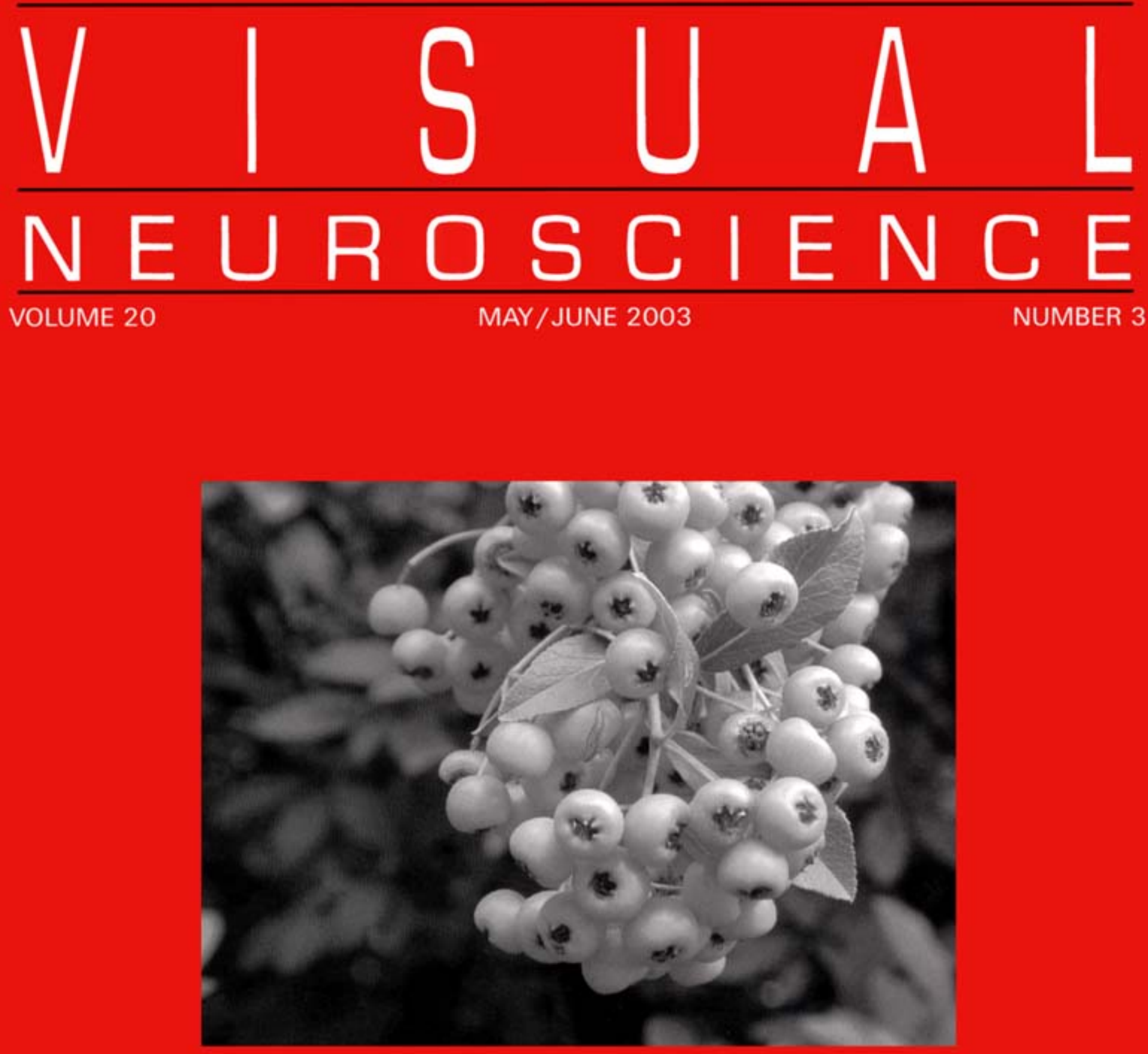

An international journal for experimental and theoretical research 


\title{
Strategies of shape representation in macaque visual area V2
}

\author{
JAY HEGDÉ* AND DAVID C. VAN ESSEN \\ Department of Anatomy and Neurobiology, Washington University School of Medicine, St. Louis
}

(Received August 19, 2002; ACCEPTED June 2, 2003)

\begin{abstract}
Contours and surface textures provide powerful cues used in image segmentation and the analysis of object shape. To learn more about how the visual system extracts and represents these visual cues, we studied the responses of V2 neurons in awake, fixating monkeys to complex contour stimuli (angles, intersections, arcs, and circles) and texture patterns such as non-Cartesian gratings, along with conventional bars and sinusoidal gratings. Substantial proportions of V2 cells conveyed information about many contour and texture characteristics associated with our stimuli, including shape, size, orientation, and spatial frequency. However, the cells differed considerably in terms of their degree of selectivity for the various stimulus characteristics. On average, V2 cells responded better to grating stimuli but were more selective for contour stimuli. Metric multidimensional scaling and principal components analysis showed that, as a population, V2 cells show strong correlations in how they respond to different stimulus types. The first two and five principal components accounted for $69 \%$ and $85 \%$ of the overall response variation, respectively, suggesting that the response correlations simplified the population representation of shape information with relatively little loss of information. Moreover, smaller random subsets of the population carried response correlation patterns very similar to the population as a whole, indicating that the response correlations were a widespread property of V2 cells. Thus, V2 cells extract information about a number of higher order shape cues related to contours and surface textures and about similarities among many of these shape cues. This may reflect an efficient strategy of representing cues for image segmentation and object shape using finite neuronal resources.
\end{abstract}

Keywords: Extrastriate cortex, Form processing, Contour analyses, Principal components analysis, Surface texture

\section{Introduction}

Natural visual scenes are typically very complex, while the neural resources of the visual system are finite, even with a billion or more neurons in the visual cortex. The visual system must extract and represent visual information within these computational constraints (see Marr, 1982; Osherson et al., 1995).

One strategy the visual system uses is to exploit information about surface textures and object boundaries, or contours, as cues to image segmentation and object shape. For example, in the image shown in Fig. 1, the curved contours, smooth and glossy curved surfaces and, in many cases, the star patterns at the tip help distinguish the berries from each other and from the other objects in the image. The manner in which berries occlude one another helps characterize local depth relationships. The angles between, and the intersections of, the stalks and leaves offer cues to their shape and spatial configuration. Psychophysical and theoretical studies indicate that the visual system indeed uses such cues in

Address correspondence and reprint requests to: David C. Van Essen, Department of Anatomy and Neurobiology, Washington University School of Medicine, 660 South Euclid Avenue, Box 8108, St. Louis, MO 63110, USA. E-mail: vanessen@v1.wustl.edu.

*Current address: Vision Center Laboratory, The Salk Institute for Biological Studies, La Jolla, CA 92037, USA. image segmentation and object recognition (see Grossberg, 1987; Wilkinson et al., 1998; Geisler \& Super, 2000).

Recent neurophysiological studies indicate that many characteristics of contours and surfaces are analyzed in early visual areas V1 and V2. Spatial characteristics known to be represented in both in V1 and V2 include selectivity for orientation, spatial frequency, length, and luminance contrast (for overviews, see Van Essen \& Gallant, 1994; Roe \& Ts'o, 1997). Selectivity for a number of additional spatial characteristics emerge or become more prominent in V2. In particular, we have previously reported that many cells in V2 are preferentially responsive to complex contours, including angles, intersection, arcs, or circles, or to non-Cartesian (hyperbolic or polar) grating patterns (Hegdé \& Van Essen, 2000).

In the present study, we have investigated how information about the characteristics of contours and gratings is represented in V2. We carried out two sets of analyses, one at the individual cell level and another at the population level. The first set of analyses was aimed at quantitatively characterizing how individual V2 cell responses vary across the overall stimulus set and by specific stimulus characteristics (shape, orientation, and size for contours; spatial frequency and orientation for gratings). This information provides useful insights into the diversity of response profiles among V2 cells and about the degree to which individual cells help represent different stimulus attributes. In the second set of analyses, we used metric multidimensional scaling (MDS) and principal 


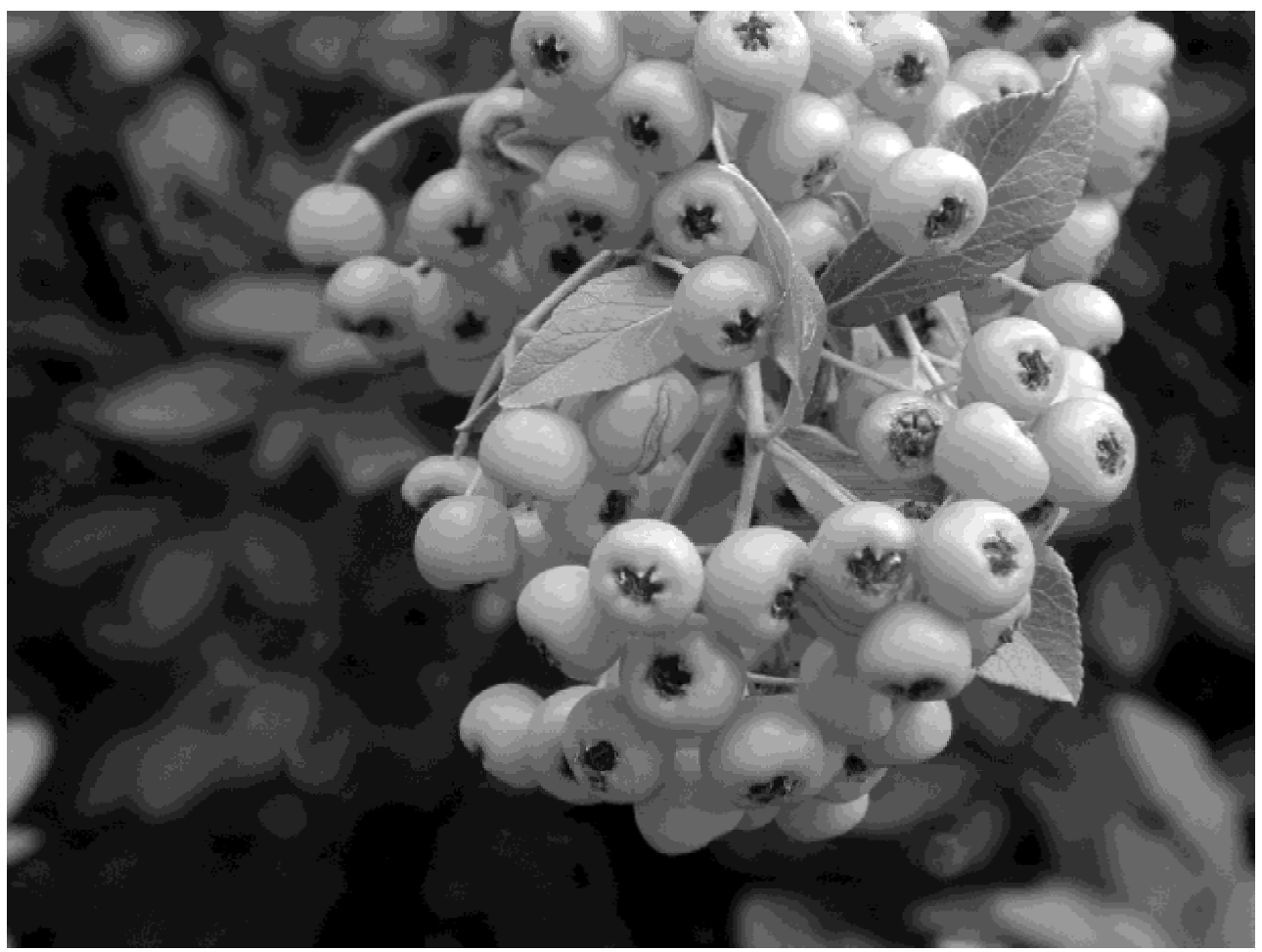

Fig. 1. A natural visual image. Contours and surface textures in the image provide useful cues to object shape and help segment the image into visual objects. The berries are characterized by their curved outlines and smooth surfaces. The stalks and leaves have relatively straight outlines and characteristic angles and intersections between the outlines. The pattern of occlusions of the contours and surfaces provide cues to the relative depth of the berries, stalks, and leaves. How does the visual system extract and represent these shape cues?

components analysis (PCA) to quantify the degree to which V2 cells as a population represent similarities between different stimuli. These methods provide an objective strategy for identifying higher order shape characteristics extracted by a given population of neurons (see, e.g. Young \& Yamane, 1992). In addition, analyzing the extent to which each principal component accounts for the overall response variation provides an objective basis for assessing the dimensionality of the neural representation (Young \& Yamane, 1992; Field, 1995; Seung \& Lee, 2000). Our analyses indicate that a relatively small number of principal components in V2 account for a high proportion of the response variance associated with complex shapes presented within the classical receptive field.

\section{Methods}

\section{Surgical and recording procedures}

The responses of single units from area V2 were recorded in awake, fixating macaque monkeys using standard procedures as described previously (Hegdé \& Van Essen, 2000). Briefly, three adult male macaques (two Macaca mulatta and one Macaca fascicularis) were used in this study. Prior to fixation training, each animal was implanted with a headpost, a scleral search coil, and an acrylic cranial patch using sterile surgical procedures. After the animal was fully trained in the fixation task, a small craniotomy ( $5 \mathrm{~mm}$ in diameter) was made through the acrylic patch over the recording site, and a recording chamber was mounted over the craniotomy. Neurophysiological recording was carried out using epoxy-coated tungsten electrodes (A-M Systems, Carlsborg, WA) with initial impedances of 3-5 M $\Omega$ (at $1 \mathrm{kHz}$ ) inserted transdurally into the cortex. All animal-related procedures used in this study were reviewed and approved in advance by the Washington University Animal Studies Committee.

\section{Stimuli}

The stimulus set consisted of 48 grating stimuli and 80 contour stimuli (Fig. 2). The grating stimuli consisted three subclasses of stimuli: (1) sinusoidal gratings, (2) hyperbolic gratings, and (3) polar (concentric and radial) gratings. The orientation and/or the spatial frequency of the gratings (or the concentric and the radial frequency, in case of polar gratings) varied systematically within each subclass (see Fig. 2).

The contour stimuli consisted of ten subclasses of stimuli, within each of which the stimuli varied in orientation and size (and also in shape in the case of subclass \#4): (1) bars; (2) 3-way intersections (tristars); (3) crosses; (4) 5- and 6-armed stars, plus circles; (5) acute angles; (6) right angles; (7) obtuse angles; (8) one-quarter arcs; (9) semicircles; and (10) three-quarter arcs. The large contour stimuli were matched in size to the cell's preferred bar length (determined qualitatively during receptive-field mapping), with the exception of large obtuse angles and one-quarter arcs, which were reduced by $50 \%$ to ensure that they stayed within the classical receptive field. In all cases, the small contour stimuli were half the size of the large contours. 
A.

\begin{tabular}{|c|c|c|}
\hline Sinusoidal & Hyperbolic & Polar \\
\hline $\begin{array}{lcc}1 & 5 & 9 \\
\| & \|\||| & \|\|||\end{array}$ & 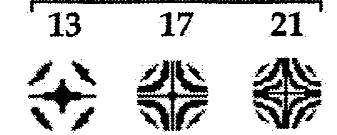 & 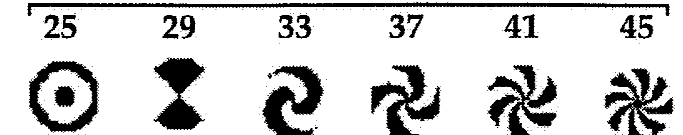 \\
\hline 10 & 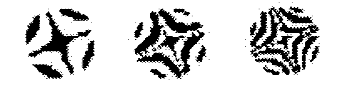 & (Q) $\times$ ( ) d \\
\hline 三 晉 & 至 & (9) * * \\
\hline U W W & 积登 & * \\
\hline
\end{tabular}

B.

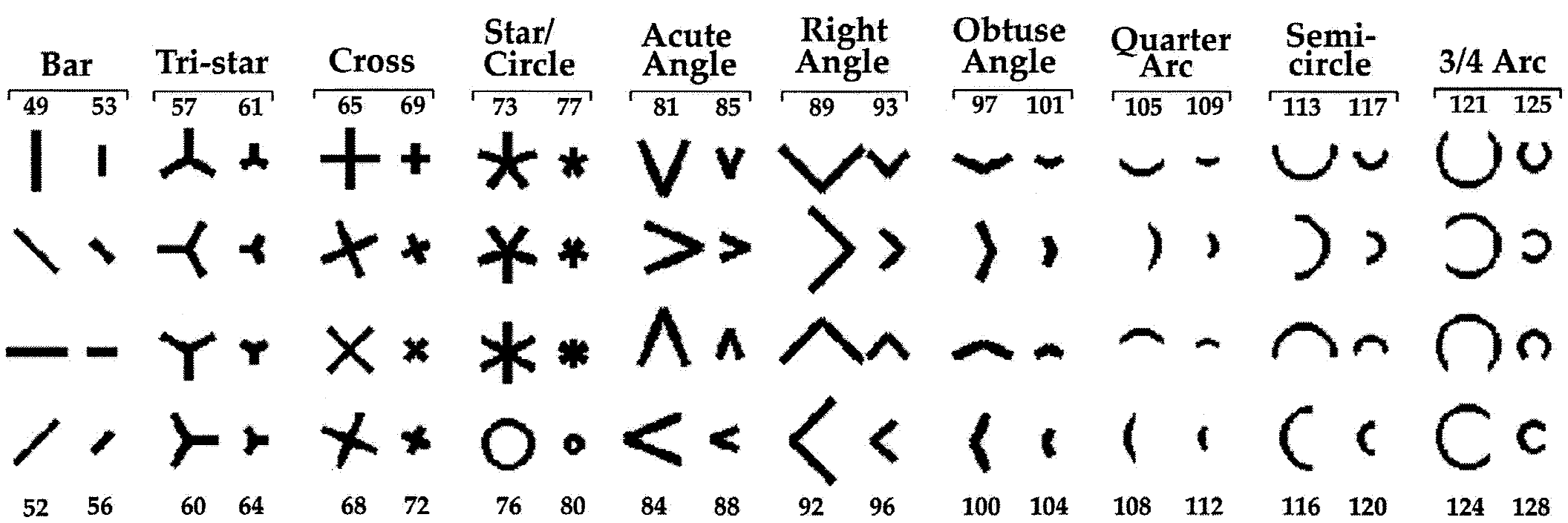

Fig. 2. The stimulus set. The stimulus set consisted of 128 stimuli, 48 of which were gratings, and the remaining 80 were contour stimuli. (A) The grating stimuli included 12 sinusoidal gratings, 12 hyperbolic gratings, and 24 polar gratings. (B) The 80 contour stimuli included bars, tristars, crosses, stars, acute angles, right angles, obtuse angles, one-quarter arcs, semicircles, and three-quarter arcs and circles. For the purposes of some of the analyses in this study, the stimuli were numbered sequentially from 1 through 128 (grating stimuli from 1 through 48 , and the contour stimuli from 49 through 128) as indicated by the numbers above and below each column of stimuli. 
As noted in the Introduction, our stimulus set was designed to explore the selectivity of V2 cells for a wide but obviously nonexhaustive set of low- and intermediate-level form cues. The grating stimuli probe the selectivity for conventional spatial frequency and orientation (sinusoids), as well as more complex textural characteristics (non-Cartesian gratings) which the visual system may use as basis functions for surface representation (Perona, 1991; Eagelson, 1992; Wilkinson et al., 1998; also see Bergen, 1991; Gallant et al., 1996). The contour stimuli were chosen to help probe the selectivity for conventional orientation (bar stimuli), along with selectivity for the angles, intersections, orientations, and curvature of visual contours, which may play an important role in image segmentation and object recognition (Rogers-Ramachandran \& Ramachandran, 1997; Geisler \& Super, 2000; Geisler et al., 2001; Sigman et al., 2001). Our choice of the grating and the contour stimuli was also motivated in part by previous reports of selectivity for these stimuli in area V4 (Gallant et al., 1993, 1996; Pasupathy \& Connor, 1999), which raised the question of whether the selectivity for these stimuli arose de novo in V4 or whether cells in lower areas of the visual hierarchy like V2 also showed selectivity to these stimuli. Practical considerations about the size of the stimulus set did not allow us address many other important shape cues (e.g. disparity, motion, etc.), and obliged us to sample many important shape characteristics (e.g. curvature) only sparsely.

\section{Visual stimulation and recording}

Single V2 cells were isolated based on both the shape and the amplitude of the waveform using a window discriminator (Bak Electronics, Germantown, MD). In most cases, we were able to isolate and maintain the waveform under study so that all and only the spikes of the given waveform fell within the window. In the small number of cases where this was not possible, the isolation window was set to minimize contamination, even at the expense of excluding some spikes of the intended waveform. The cell's receptive-field boundaries were mapped using mouse-driven bar and grating stimuli on the computer's monitor. The cell's preferred bar parameters, including preferred length, width, color, and orientation, were also determined. We tested all cells with mappable receptive fields which met the isolation criteria described above. Prior to recording from the cell, the stimulus set was reoriented according to the cell's preferred orientation (see legend to Fig. 2). The line width of contour stimuli was set at the cell's qualitatively determined preferred bar width. The grating stimuli had a spatial frequency of 2, 4, or 6 cycles per receptive-field diameter and a Michelson contrast of 1.0. All stimuli were presented in the cell's preferred color, selected during the manual mapping from a palette of seven colors with varying luminances (red, $1.18 \mathrm{~cd} / \mathrm{m}^{2}$; green, $5.13 \mathrm{~cd} / \mathrm{m}^{2}$; blue, $0.51 \mathrm{~cd} / \mathrm{m}^{2}$; aqua, $5.70 \mathrm{~cd} / \mathrm{m}^{2}$; pink, $1.82 \mathrm{~cd} / \mathrm{m}^{2}$; yellow, $7.02 \mathrm{~cd} / \mathrm{m}^{2}$; and white, $7.76 \mathrm{~cd} / \mathrm{m}^{2}$; all measured using Tektronix J17 photometer, Beaverton, OR). For all cells, the stimuli were presented against the same uniform gray background, the luminance of which was set at $3.71 \mathrm{~cd} / \mathrm{m}^{2}$ to maximize fixation performance of the animal and minimize light adaptation by the cells (see Wandell, 1995). This meant that both luminance and stimulus-background contrast varied from one cell to the next, depending on the preferred color. The proportion of cells which preferred colors with lower versus higher luminance than the background did not significantly differ from that expected from chance (two-tailed binomial probability test, $P>0.05$ ).
Each stimulus was presented in each of three jitter positions centered $12.5 \%$ of the receptive-field diameter away symmetrically around the receptive-field center. Stimuli were presented sequentially for $300 \mathrm{~ms}$ each with a 300-ms interstimulus interval within the classical receptive field while the animal fixated within a window of 0.5 -deg radius for a liquid reward. Up to six stimuli were presented per trial in this fashion. Only the data from the trials throughout which the animal maintained fixation within the fixation window were used in this study. A total of 196 cells were recorded, 122 from animal \#1 and 63 cells from animal \#3. Recording from animal \#2 was discontinued after 11 cells because of poor fixation performance.

\section{Analysis of data}

Data analyses were carried out using the statistical utility S-Plus (Statsci, Inc., Seattle, WA) or custom-written C language software. The response to each stimulus was averaged from the net firing rate from 12 repetitions of the stimulus, with four repetitions at each jitter position ( 9 repetitions, with three repetitions at each jitter position, for 62 cells). The net firing rate was calculated for each presentation of the given stimulus by subtracting the background rate from the corresponding visually evoked response. The background firing rate was calculated using a 80-100 ms window (mean, $94 \mathrm{~ms}$; median, $95 \mathrm{~ms}$ ) immediately preceding the stimulus onset. The evoked response was calculated using a $80-285 \mathrm{~ms}$ time window (mean duration, $169 \mathrm{~ms}$; median, $150 \mathrm{~ms}$ ) starting 20-80 ms after the stimulus onset (mean onset, $27 \mathrm{~ms}$, median, $30 \mathrm{~ms}$ ), during which the overall firing rate of the cell (across all repetitions of all stimuli) remained above background levels. Each cell included in this study had at least one stimulus for which the evoked response differed from the background response at a significance level of $P<0.05$ (two-tailed $t$-test with Bonferroni correction for multiple comparisons). Of a total of 196 cells recorded from the three animals, 180 cells (108 cells from animal \#1, 11 cells from animal \#2, and 61 cells from animal \#3) passed this test and were included in this study.

\section{Tests of significance}

Conventional parametric tests of significance were used where appropriate. In most cases, however, we used randomization analysis. A test of significance using randomization consists of determining whether the value of a user-defined test statistic calculated from the actual data differs significantly from the distribution of the same test statistic calculated from randomized data (see Manly, 1991 for an overview). For each test, an appropriate test statistic was first calculated using the actual neural response data. The data were then randomized in a manner appropriate for the given test, and the test statistic was recalculated using the randomized data. The randomization process was repeated $10^{6}$ times $\left(10^{3}\right.$ times in case of MDS analyses). The proportion of times the randomized test statistic exceeded the actual test statistic constituted the onetailed probability $P$ that the actual test statistic was significantly greater than random.

In cases involving multiple comparisons, we adopted a stringent approach of using the Bonferroni correction $(\alpha=0.05 / \mathrm{n}$, where $\alpha$ is the probability of Type I error and $n$ is the number of comparisons; see Huberty \& Morris, 1989; also see Savitz \& Olshan, 1995; Thompson, 1998).

\section{Indices of response modulation}

Two sets of indices were used to characterize various aspects of stimulus selectivity of V2 cells. In either case, larger values of a 
given index represented correspondingly greater stimulus selectivity by that measure. One set, the modulation indices, measured the modulation, or variation, of a given cell's responses across a given subset of stimuli above random noise levels using the conventional $F$ ratio. To calculate a given modulation index, we first calculated the $F$ ratio of the cell's responses to the given set of stimuli, given by $F=M S_{\text {between }} / M S_{\text {within }}$, where $M S_{\text {between }}$ is the stimulus-tostimulus variance (or, equivalently, the between-stimulus mean squares), and the $M S_{\text {within }}$ is the average trial-to-trial variance (see Snedecor, 1934; Brase \& Brase, 1995). We next randomized the responses across the stimuli and recalculated the $F$ ratio. The value of a given modulation index was defined as the $F$ ratio calculated from the actual data divided by the average $F$ ratio from the randomization rounds. The various modulation indices differed from each other only in terms of the stimuli across which the response modulation was measured.

The second set, the stimulus selectivity indices (SSI), measured the peakedness of the cell's response profile (i.e. responses of the cell to a given [sub]set of stimuli) and had the general form [1 $\left.-\left(R_{\text {mean }} / R_{\text {peak }}\right)\right]$, where $R_{\text {mean }}$ is the average response of the cell to the given subset of stimuli and $R_{\text {peak }}$ is the response of the cell to its most effective stimulus from among the given subset of stimuli. The various stimulus selectivity indices differed from each other only in terms of the stimuli involved.

\section{Analysis of response correlations}

To analyze patterns of response correlation across the population, we used MDS and PCA. Both are well-established and widely used multivariate statistical techniques for analyzing complex, high-dimensional data (for overviews, see Kruskal \& Wish, 1978; Dunteman, 1989; Kachigan, 1991). As the input to MDS or PCA, we used a $128 \times 128$ correlation matrix, each element of which represented the correlation coefficient of the responses of the V2 cells (averaged across trials, but not normalized) to a given pair of the 128 stimuli. We used the correlation coefficient rather than the coefficient of covariance, because the former is scale invariant, measures response similarities independent of the absolute firing rates, and is commonly used for this purpose (see Young \& Yamane, 1992; Oja, 1995; Simoncelli \& Olshausen, 2001).

MDS plots the data so that the distances between the data points, in our case the stimuli, represents the similarity of the responses of V2 cells to the stimuli. MDS can be implemented using many slightly different algorithms, all of which produce qualitatively similar results (see Kruskal \& Wish, 1978; Cox \& Cox, 1994); we used the classical algorithm (S-Plus routine $\mathrm{cmd}$ scale), which uses spectral decomposition of the input matrix (see Kruskal \& Wish, 1978; Cox \& Cox, 1994). The algorithm begins with an arbitrary placement of the stimuli and iteratively shifts the stimuli in order to reduce the distortion (or "stress") between the interstimulus distances and the original similarities. Some residual stress is to be expected when reducing a high-dimensional data into a two-dimensional (2D) format. However, the algorithm finds the best representation of similarities with the least amount of distortion, so that stimuli which elicit dissimilar responses are dispersed from each other and the stimuli which elicit similar responses are clustered together.

\section{Analysis of MDS clusters}

We used randomization analysis to determine whether the clustering of stimuli, if any, in a given MDS plot was significantly nonrandom. The test statistic was the $D$ ratio, which is directly analogous to the $F$ ratio (see below). An MDS plot was first generated using the original $128 \times 128$ correlation matrix described above. Clusters of data points were provisionally identified from a visual examination of the plot and the $D$ ratio, defined as the variance of the between-cluster distances divided by the mean variance of within-cluster distances, was calculated. The correlation matrix was then randomized and an MDS plot was generated from the randomized matrix. The $D$ ratio was calculated for this MDS plot using the original composition of the clusters. The clustering in the original matrix was considered significantly nonrandom if the $P$ value was less than 0.05 .

\section{Cophenetic correlation}

Cophenetic correlation is a method of calculating the correlation coefficient between paired matrices or other high-dimensional data (for overviews, see Sokal \& Rohlf, 1962; Sneath \& Sokal, 1973). To calculate the cophenetic correlation between a given pair of stimulus plots, such as those generated by MDS, we first calculated all pairwise distances among the 128 stimuli in each plot. This resulted in two paired vectors, one from each plot, each containing $128^{2}$ elements. The cophenetic correlation coefficient $r_{\mathrm{C}}$ between the two 2D plots is the conventional correlation coefficient between the two paired vectors. Like the conventional correlation coefficient, the values of $r_{\mathrm{C}}$ vary from 1.0 (perfect correlation) to 0.0 (no correlation) to -1.0 (perfect anticorrelation).

PCA (S-Plus routine princomp) simplifies complex, highdimensional data by identifying a small number of factors that underlie global patterns in the data and determining the extent to which each factor, or principal component, "explains" the data. In a manner analogous, but not identical, to multiple linear regression, PCA linearly transforms an original set of variables into a smaller set of independent (i.e. uncorrelated) variables that represent most of the information in the original set of variables. We used this technique to assess whether the V2 population response could be adequately accounted for by a small number of response patterns. Composites of the loadings were constructed using the method of Turk and Pentland (1991). Briefly, to construct the composite for a given principal component, each stimulus was rendered using a grayscale value that represented its loading on the given component, so that loadings of $1.0,0$, and -1.0 resulted in, respectively, a stimulus that was white, neutral gray (i.e. background), or black in color. The stimuli were then averaged across space so that the composite for a given principal component represented the weighted average of the loadings on the individual stimuli.

\section{Results}

\section{The diversity of $V 2$ response profiles}

The response profiles of individual V2 cells were often complex and differed considerably from one cell to the next, as illustrated by the exemplar cells in Fig. 3 (also see Fig. 1 of Hegdé \& Van Essen, 2000). The cell shown in Fig. 3A was sharply tuned for shape. It responded maximally to the large right angle at $180 \mathrm{deg}$ (82 spikes/s, averaged across repetitions; third row). This response was considerably larger than the sum of the responses to bars at $45 \mathrm{deg}$ and $135 \mathrm{deg}$, suggesting a strongly nonlinear summation. The second most effective stimulus, a large acute angle at $270 \mathrm{deg}$ (fourth row), elicited about two-thirds of the maximal response (57 spikes/s). All other stimuli, including the remaining right angle 


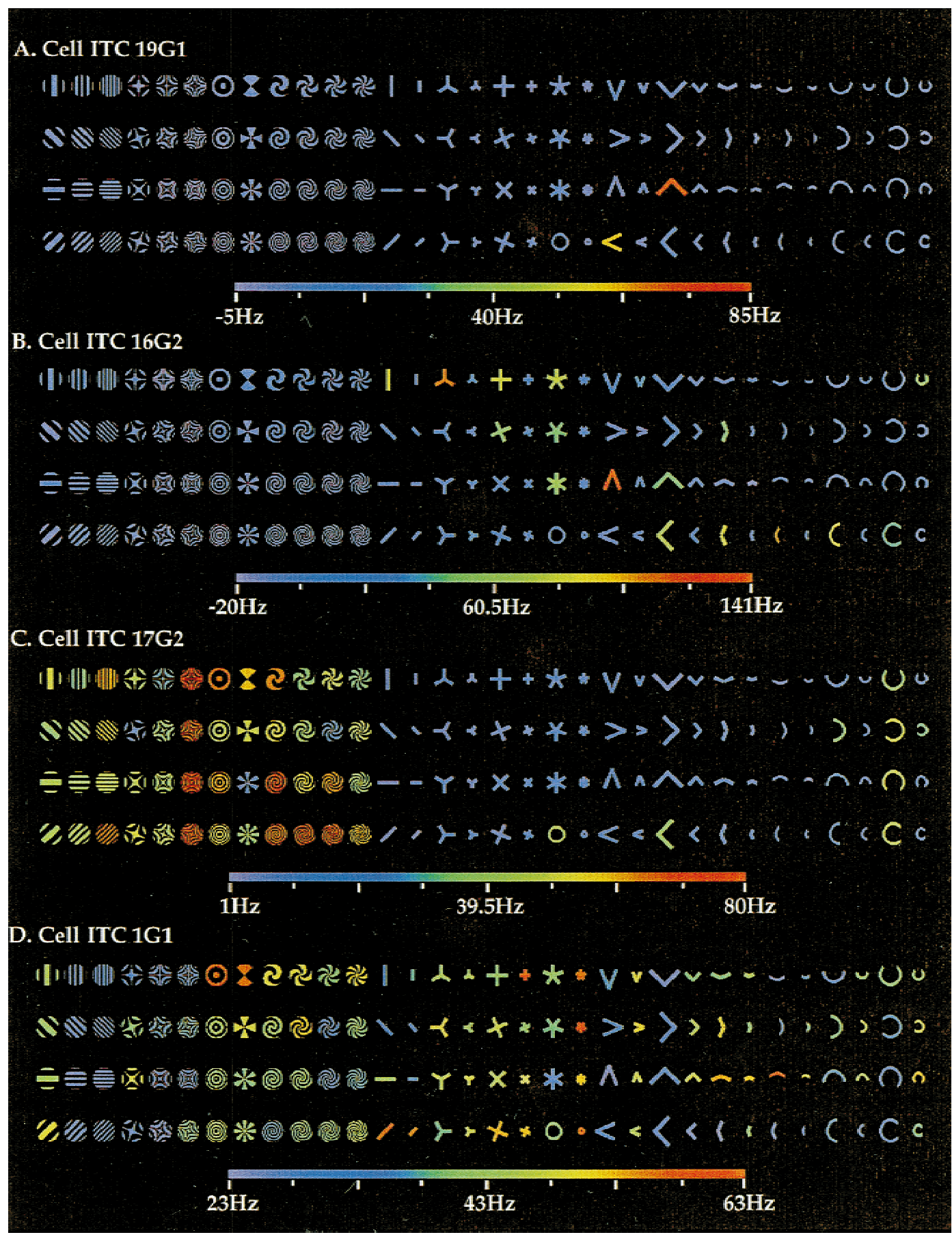

Fig. 3. The diversity of V2 response profiles. Each panel illustrates the responses of a single V2 cell to the stimulus set. The color of each stimulus represents the net responses of the cell to the stimulus averaged across trials according to the color scale below each panel. In each panel, stimulus orientations are normalized so that the preferred orientation, as determined during the manual mapping of the receptive field, is shown as vertical. Occasionally, as in panel D, the quantitatively determined preferred orientation differed from that estimated during manual plotting. The veridical preferred orientations determined during manual mapping for cells shown in panels A-D were 90, 135, 30, and 60 deg, respectively. The trial-to-trial variations in the response (not shown) were generally small relative to the mean responses. Across all stimuli and all 180 cells in our population, the average SEM of responses was $11.3 \%$ of the mean responses. The response variances associated with systematic variations in the spatial placement of the stimulus within the receptive field were also generally low (not shown).

or acute angle stimuli, elicited less than one-eighth of the maximal response (average response, 6 spikes/s).

Most V2 cells were considerably more broadly tuned than the cell in panel A. For the cell shown in panel B, the most effective stimulus was the large acute angle at $180 \mathrm{deg}$ (third row; 141 spikes/s), but the cell responded well to many other angles, intersections, arcs, and its preferred bar. The cell's responses were substantially modulated by the shape characteristics of these con- 
tour stimuli, such as the stimulus type (cf. acute $v s$. right angles), orientation (cf. large crosses at $0,22.5,45$, and $67.5 \mathrm{deg}$ ), and size (cf. large $v s$. small three-quarter arcs), indicating that the responses of the cell conveyed information about the shape characteristics of many of the contour stimuli. Most of the effective stimuli contained orientation components near the cell's preferred orientation (e.g. large tristar at 0 deg [top row], large five- and six-armed stars [rows 1-3], and the large obtuse angle at $270 \mathrm{deg}$ [fourth row], and the large one-quarter arc at $270 \mathrm{deg}$ [fourth row]). However, the cell responded poorly to the large acute angle at $0 \mathrm{deg}$ (top row), even though it contained the same orientation components as the cell's most effective stimulus.

The cell shown in Fig. 3C responded well to grating stimuli in general, with the high-frequency polar grating (fourth row) eliciting the maximal response (80 spikes/s), and 14 other grating stimuli, mostly at the lower spatial frequencies, eliciting at least three-quarters of this response. Although the cell responded poorly to most contour stimuli (mean response of 17 spikes/s across all contour stimuli), it responded relatively well to the large circle and three-quarter arcs (mean response of 48 spikes/s to these five stimuli). Also, the cell responded well to many polar gratings on the one hand and to the large circle and three-quarter arc stimuli on the other, suggesting that curvature selectivity may have contributed strongly to this cell's response profile. The cell in panel D responded maximally to a small five-armed star (63 spikes/s; second row). However, many stimuli (16 grating stimuli and 41 contour stimuli) elicited more than two-thirds of the maximal response of the cell, and the least effective stimulus, a large acute angle at 180 deg (third row), elicited 23 spikes/s above background. Also, the cell generally responded better to small contour stimuli than to their larger counterparts (cf. small vs. large acute angles and three-quarter arcs).

\section{The population average response}

To gauge the response of $\mathrm{V} 2$ cells as a population to the stimulus set, we calculated the normalized average population response to each stimulus. Each cell's responses to all 128 stimuli were normalized so that the cell's responses to its most and the least effective stimuli were 1.0 and 0 , respectively, thereby ensuring that each cell contributed to the same extent to the population average. The normalized responses were then averaged across all 180 cells.

Fig. 4A shows the average responses of the population to individual stimuli. The top four stimuli, each of a different subtype [including the preferred bar (top row), Cartesian grating (top row), a concentric grating (top row), and a large acute angle at $180 \mathrm{deg}$ (third row)] elicited similar responses $(0.50,0.50,0.47$, and 0.47 , respectively). Nearly half the stimuli $(56 / 128,44 \%)$, including nearly four-fifths of the grating stimuli $(38 / 48,79 \%)$, elicited more than three-quarters of the maximal response (i.e. $>0.375$ ). The least effective stimulus for the V2 cell population, a small onequarter arc at $0 \mathrm{deg}$ (top row), elicited nearly half of the maximal response $(0.23)$. Thus, the population average responses varied over a relatively narrow range. In addition, all stimuli showed substantial cell-to-cell-variation of response (Fig. 4B) (maximum SD [preferred bar, top row], 0.30; minimum SD [small right angle at $0 \mathrm{deg}$, top row], 0.17). Together, these results indicate that no single stimulus was consistently effective or ineffective across the population of V2 cells.

The population average responses demonstrate that V2 cells were generally more responsive to the grating stimuli as a group (mean response, 0.44; range, 0.50-0.34) than to the contour stim- uli (mean responses, 0.36; range, 0.50-0.23). Also, larger contour stimuli were relatively more effective (mean response, 0.38 ) than the small contour stimuli (mean response, 0.27).

Together, the exemplar cells and the population response suggest that the responses of $\mathrm{V} 2$ cells are modulated by many different shape characteristics. In what follows, we first quantitate the degree to which the various shape characteristics of the stimuli modulate the responses of individual V2 cells. We then study how $\mathrm{V} 2$ cells as a population represent the shape information.

\section{Individual V2 cells carry information about many shape characteristics}

\section{Response modulation across all stimuli}

To measure the extent to which the responses of individual V2 cells were modulated by the stimulus set as a whole, we used two indices, each addressing a different aspect of the cell's response profile. To determine whether the modulation of the given cell's responses across all 128 stimuli was larger than expected by trial-to-trial variations in response, we calculated its overall modulation index, $O M I$ (see Methods). V2 cells had an average $O M I$ value of 4.03 , indicating that, on an average, the overall response of V2 cells was modulated about four-fold above the level expected from random (Fig. 5A). The response modulation was significant (randomization analysis, $P<0.05$ ) for about ninetenths of the cells $(163 / 180,91 \%$; filled bars in Fig. 5A). The median OMI value was 2.72 , indicating that the responses were modulated greater than 2.72-fold above random levels for half of the population.

To measure the degree of selectivity for the most effective stimulus, we calculated a stimulus selectivity index (SSI), given by [1 $-\left(R_{\text {mean }} / R_{\text {peak }}\right)$ ], where $R_{\text {mean }}$ is the average response of the cell to all 128 stimuli and $R_{\text {peak }}$ is the response of the cell to its most effective stimulus. SSI values of 0.50 and 0.75 denote that the response to the cell's most effective stimulus was, respectively, two and four times larger than the cell's average response to the stimulus set as a whole. The exemplar cells shown in Figs. 3A and $3 \mathrm{D}$, for instance, had SSI values of 0.93 and 0.32 , respectively. The average $S S I$ value of V2 cells was 0.69 , indicating that the response of an average V2 cell to its most effective stimulus was about three-fold larger than its average response to all stimuli (Fig. 5B). The SSI values were poorly correlated with the $O M I$ values $\left(r_{S S I, O M I}=0.26\right)$, indicating that the two indices measured relatively independent aspects of the response profiles. The degree of selectivity for the preferred stimulus as measured by the SSI was significantly higher than chance (randomization test, $P<0.05$ ) for about four-fifths of the cells (145/180, 81\%; filled bars in Fig. 5B). The results were qualitatively similar when sparseness (see Rolls \& Tovee, 1995; Vinje \& Gallant, 2000; Friedrich \& Laurent, 2001), instead of SSI, was taken as a measure of the peakedness of the response profile (data not shown).

Altogether, the distributions of the two indices show that V2 cells were able to convey significant shape information using a diversity of coding styles, varying from local coding to broad tuning. Importantly, no pronounced clustering of tuning characteristics was evident by either index.

\section{Responses to gratings vs. contours}

For many V2 cells, the response profiles to gratings and contours differed substantially from each other. For instance, the exemplar cell shown in Fig. 3C responded well to, and was broadly 


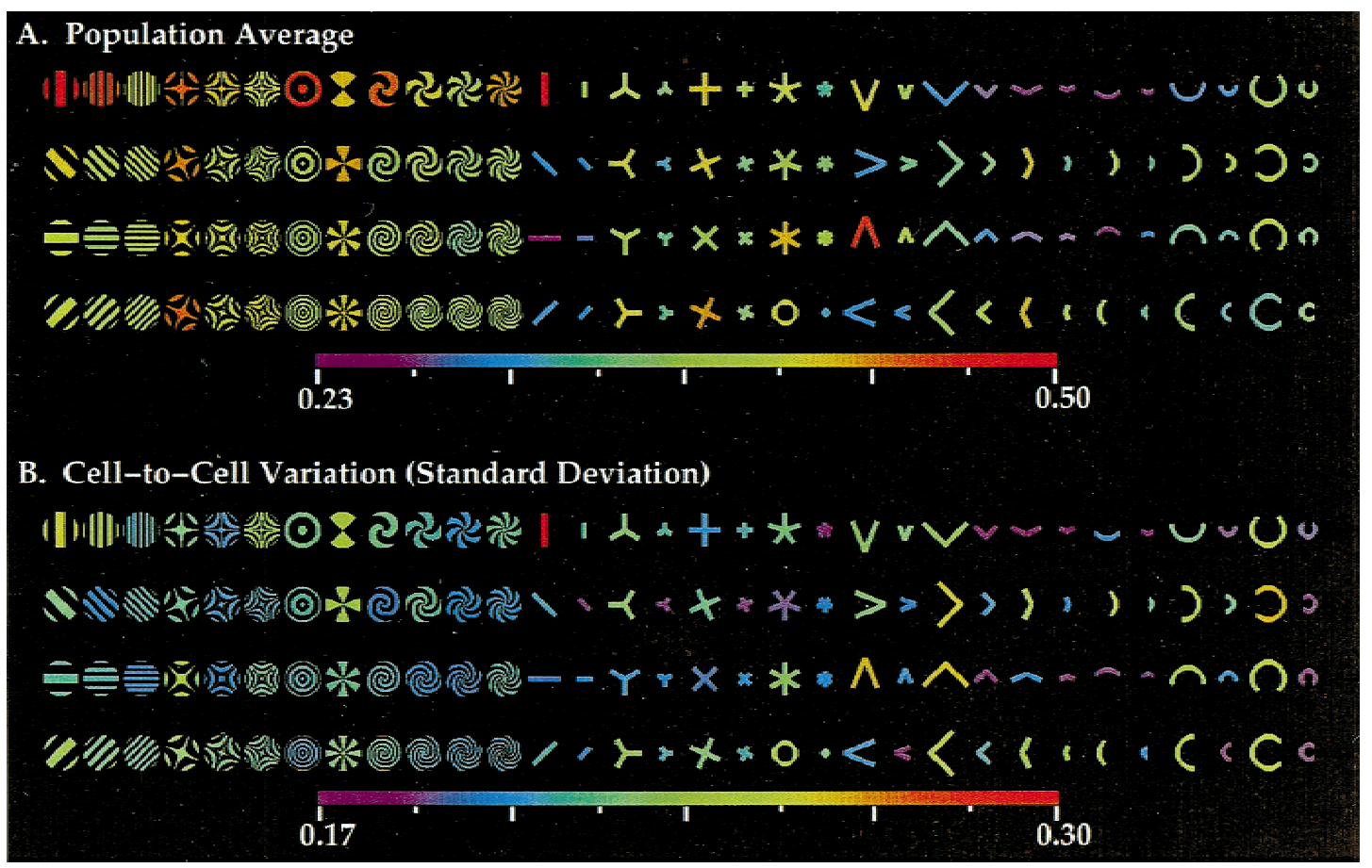

Fig. 4. The population response. (A) The normalized average response of all $180 \mathrm{~V} 2$ cells to the stimulus set. The maximum and the minimum response from each cell were normalized to 1.0 and 0 , respectively, and averaged across all 180 cells. The resulting population averages ranged from 0.5 to 0.23 , according to the color scale shown at the bottom. (B) The cell-to-cell variation of the population response, measured as the absolute (i.e. non-normalized) standard deviation of the normalized responses to each stimulus.

tuned for, grating stimuli as a class, but responded much more selectively to contour stimuli. Conversely, the exemplar cell shown in Fig. 3B was largely unresponsive to grating stimuli as a class, but responded well to many contour stimuli.

To determine the extent to which V2 cells as a population responded differentially to the grating versus contour stimuli in our stimulus set, we carried out two analyses, one involving peak responses and the other involving mean responses. Fig. 6A shows the peak response of each cell to contour stimuli (i.e. the response to the cell's most effective contour) plotted against its peak grating response. For about two-thirds of the cells $(118 / 180,66 \%)$, the peak contour response exceeded the peak grating response (the

A.

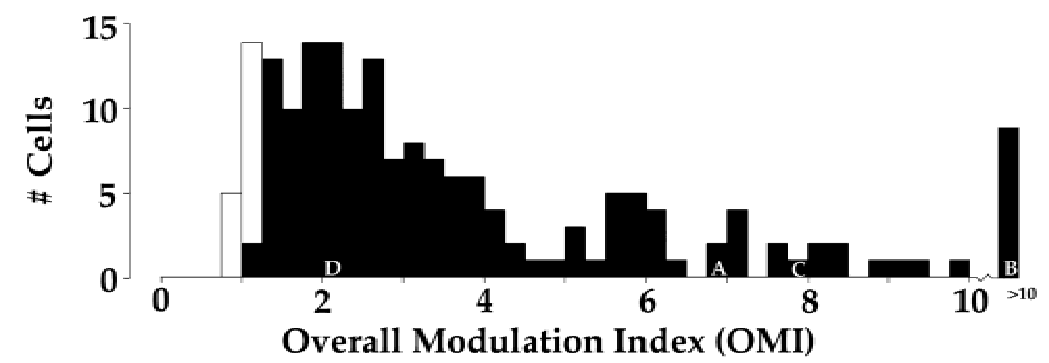

B.

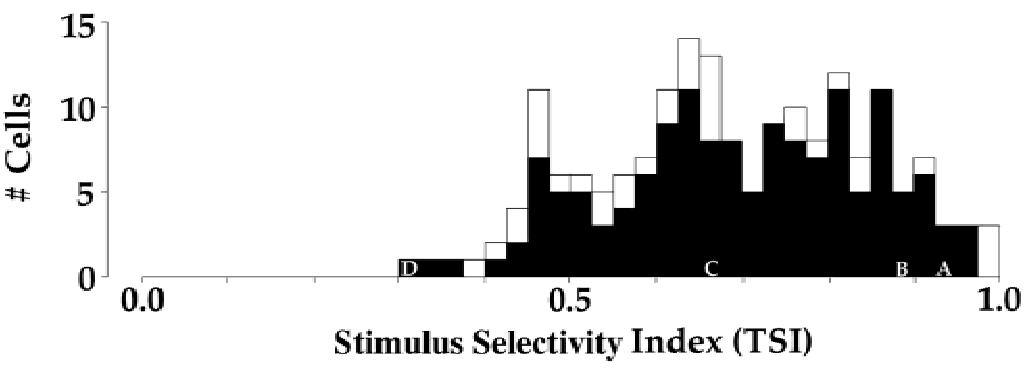

Fig. 5. Response of modulation across all 128 stimuli for individual V2 cells. For each cell, the modulation of responses by the stimulus set as a whole was measured using two different indices. The distribution of the resulting index values is shown here in histogram form. (A) Overall modulation index (OMI). (B) The stimulus selectivity index $(S S I)$. In either panel, the filled bars represent those cells for which the response modulation as measured by the given index response was significantly above random $(P<0.05)$ as determined by the randomization of spike counts across stimuli (see Methods). The cells with $P>0.05$ are denoted by open bars. In this and the subsequent figures, the exemplar cells in Figs. $3 A-D$ are indicated by the appropriate letters. 

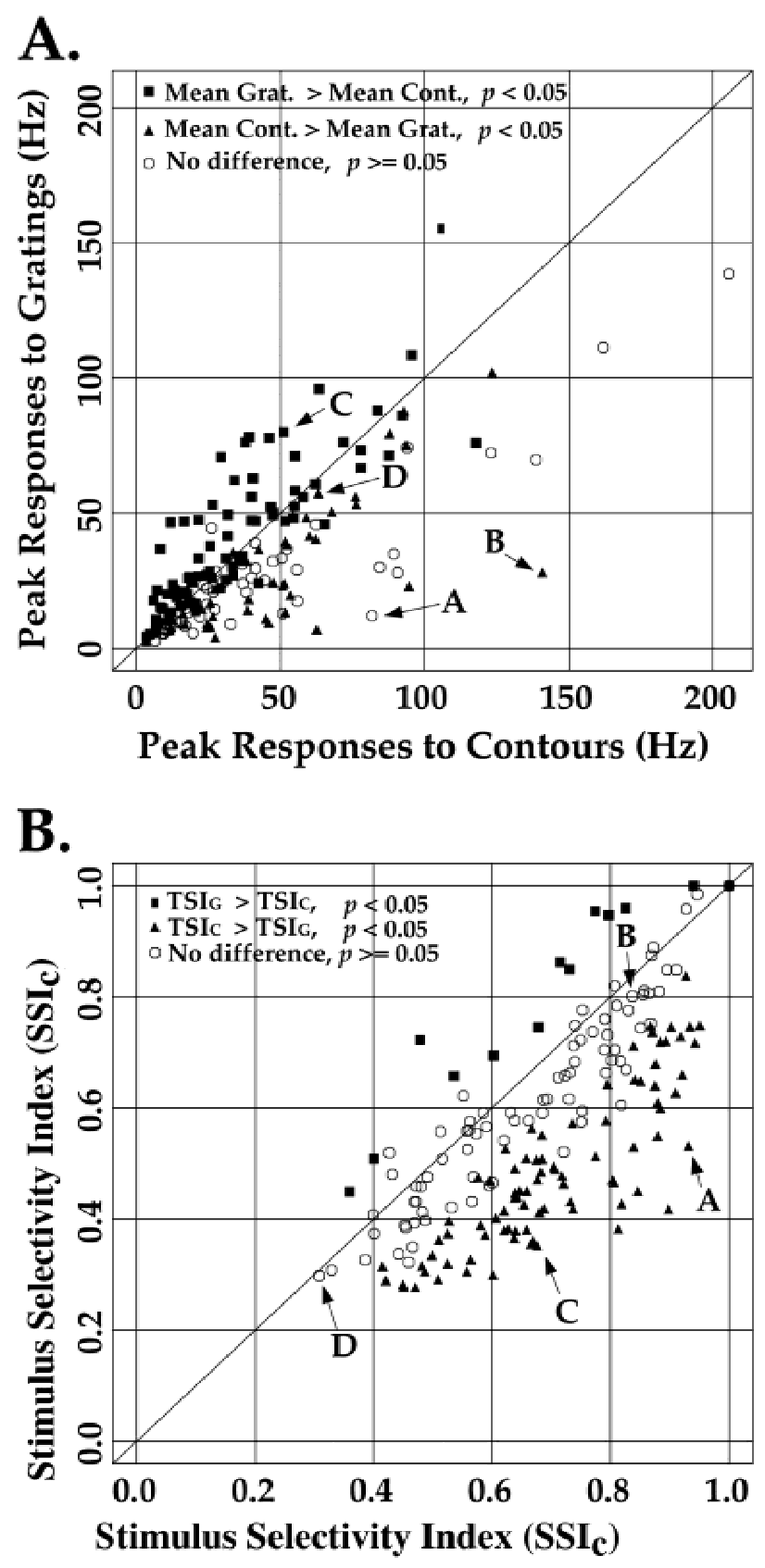

Fig. 6. Comparisons of V2 cell responses to contour vs. grating stimuli. (A) Analyses of both peak and the mean responses are represented in this panel. The $x$ and $y$ coordinates of each cell denote the peak responses of the cell to the contour and the grating stimuli, respectively. The plotting symbols denote the results of a one-tailed $t$ test comparing the mean responses of each cell to contours vs. gratings (and vice versa). Filled squares denote cells for which the responses to the 48 grating stimuli were significantly larger than the responses to the 80 contour stimuli $(P<0.05)$. Filled triangles denote cells for which the responses to the contour stimuli were larger than the responses to the gratings $(P<0.05)$, and open circles denote cells which showed no preference $(P>0.05)$. (B) The stimulus selectivity indices for the contour and the grating stimuli $\left(S S I_{\mathrm{C}}\right.$ and $S S I_{\mathrm{G}}$ indices, respectively) are plotted against each other for each cell. Outlier cells with index values of $>1.0$ were normalized to 1.0. Filled triangles denote the cells for which the $S S I_{\mathrm{C}}$ values were significantly larger than the $S S I_{\mathrm{G}}$ values (randomization test, $P<0.05$ after Bonferroni correction). The cells for which $S S I_{\mathrm{G}}$ values were significantly larger than the $S S I_{\mathrm{C}}$ values $(P<0.05)$ are shown as filled squares. The cells which did not show a significant preference $(P>0.05)$ are shown as open circles. cells below the diagonal); this preference was statistically significant (one-tailed $t$-test, $P<0.05$, after Bonferroni correction) for about one-third of these cells $(43 / 118,36 \%$, or $24 \%$ of the total; data not shown). For the remaining cells, whose peak grating response exceeded the peak contour response $(62 / 180,34 \%)$, the preference was statistically significant for about one-third (18/62; $29 \%$, or $10 \%$ of the total). However, the greater incidence of contour-preferring cells may simply reflect a sampling bias, owing to the fact that there were more contour stimuli than grating stimuli (80 vs. 48).

We found more pronounced difference between the two stimulus classes of stimuli in the mean response analysis, which compared the responses of each cell averaged across the 48 grating stimuli to the responses averaged across the 80 contour stimuli. The results are denoted by the plotting symbols in Fig. 6A. For nearly half of the cells $(81 / 180,45 \%)$, the average grating response was significantly larger (using a one-tailed $t$-test) than the average contour response $(P<0.05$; filled squares $)$, whereas average contour responses significantly exceeded the average grating responses for only about a quarter of the cells $(44 / 180,24 \%$; filled triangles). Nearly one-third of the cells (44/180, $31 \%$; open circles) showed no significant preference. The response of V2 cells to gratings was on average 1.62-fold larger than the response to contour stimuli.

To compare the sharpness of tuning of V2 cells to grating versus contour stimuli, we calculated the sharpness of tuning index separately for the grating and the contour stimuli $\left(S S I_{\mathrm{G}}\right.$ and $S S I_{\mathrm{C}}$, respectively; see Methods). A great majority of V2 cells (151/180, $84 \%$ ) were more broadly tuned to gratings than to contours (Fig. 6B; cells below the diagonal), and this difference was significant in about half $(47 \%)$ of the total population $\left(S S I_{\mathrm{C}}>S S I_{\mathrm{G}}\right.$, randomization test, $P<0.05$ after Bonferroni correction). By contrast, the tuning for the grating stimuli was sharper for only $16 \%$ of the cells (29/180), and significantly so for only $7 \%$ of the total population. Together, these results indicate that V2 cells responded better on average to the grating stimuli in our stimulus set, but were more selective for the contour stimuli. The magnitude of this bias depends, of course, on the particular choice of stimuli and might be different (or even nonexistent) for other reasonable choices for stimulus sets.

\section{Response modulation by stimulus size}

Most V2 cells responded better to large contour stimuli than to small contour stimuli (Fig. 4A; also see Figs. 3A \& 3B). Aside from the differences in overall responsiveness, it is of interest to know whether individual cells respond similarly to the large versus the small version of each contour shape, that is, whether they show size invariance across the two sizes sampled. To address this issue, we calculated the correlation coefficient $r$ between the cell's responses to large versus small contour stimuli. Because the issue is primarily of interest for cells that were substantially responsive to both large and small contours, we identified a subset of 77 cells (filled bars in Fig. 7) meeting this criterion (see legend to Fig. 7). The average correlation for this subgroup of 77 cells was 0.51 , substantially greater than the value of 0.32 for the population as a whole. These results suggest that many, but not all, V2 cells show considerable size invariance for the contour stimuli tested.

We also measured the response modulation within each of the 14 stimulus subclasses using the $F$ ratio-based modulation indices. These analyses showed that many V2 cells conveyed information about each of the shape characteristics that varied within each stimulus subclass, including orientation, spatial frequency, or size of various stimuli (data not shown). 
Responsive to both large and small contours

Unresponsive to large and/or small contours

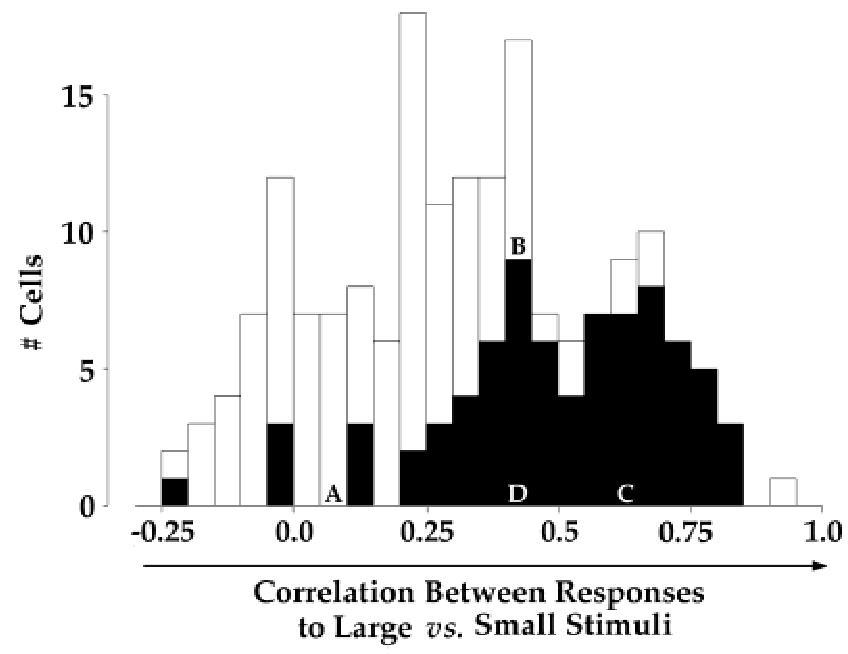

Fig. 7. Correlation of responses to large vs. small contour stimuli. The histogram shows the distribution of correlation coefficients comparing the responses of each cell to the large vs. small contours. The filled bars denote cells which conveyed significant information about both large and small contours, by virtue of passing five separate randomization tests at $P<$ 0.05 . The tests measured response modulation across (1) large vs. small contours, (2) large contours alone, (3) small contours alone, and compared (4) evoked responses vs. background responses to large contours, and (5) evoked responses $v s$. background responses to small contours. The first three tests used the $F$ ratio as the test statistic; the last two tests used the $t$ statistic. Open bars represent cells with $P>0.05$ for one or more of the tests.

\section{Population-wide patterns of response}

From the results presented thus far, it is unclear whether the response profiles of V2 cells are mutually unrelated or whether there are meaningful similarities among the response profiles of various V2 cells. We therefore studied the V2 population response for global patterns of response across all cells. To do this, we constructed a $128 \times 128$ correlation matrix of the population response, each element of which represented the correlation coefficient of the responses of all V2 cells to a given pair of the 128 stimuli (see Fig. 8 for details). For example, two geometrically similar spirals (stimuli 44 and 48 in Fig. 2) elicited strongly correlated responses across the population (correlation coefficient $r=0.72$ ), as shown in Fig. 8A, whereas two geometrically disparate stimuli (spiral $47 \mathrm{vs}$. bar 49) elicited poorly correlated responses $(r=-0.08)$ as shown in Fig. 8B. Other stimulus pairings, such as the arc (stimulus 114) and the chevron (stimulus 98) shown in Fig. 8C had intermediate correlation values $(r=$ 0.12 ). These correlation values were used to generate an overall correlation matrix, which is shown in its entirety in Fig. 8E and for a small portion in Fig. 8D in order to illustrate the contributions of the exemplar correlation values in panels $\mathrm{A}-\mathrm{C}$.

We analyzed this correlation matrix using both multidimensional scaling (MDS) and principal components analysis (PCA). The MDS analysis reveals clusters of stimuli that tend to elicit correlated responses across the population. The PCA analysis provides insights regarding the dimensionality needed to account for the diversity of response profiles in the population.
Patterns of response correlation: MDS analysis

The MDS plot derived from the correlation matrix reveals three large clusters, each shown in a different color in Fig. 9. The 48 grating stimuli were grouped in a single, well-defined cluster (stimuli shown in red). Thus, grating stimuli as a class elicited similar (i.e. correlated) responses from V2 cells, consistent with the results described earlier (see Fig. 6). The 80 contour stimuli were more dispersed, but were identifiable as two relatively loose clusters. One cluster (stimuli shown in green) contained 17 large angles and arcs (including all the large right angles, semicircles, circle, and three-quarter arcs and three of the four large acute angles); the other cluster (stimuli shown in blue) contained the remaining contour stimuli. In addition, the arrangement of stimuli within each cluster does not appear random. Within the three main clusters, many smaller subclusters of stimuli were discernible, such as the large three-quarter arcs within the green cluster, and small intersections within the blue cluster (dashed ellipses in Fig. 9). Within the grating stimulus cluster, radial gratings were generally segregated from the concentric gratings.

To assess the statistical significance of these three subjectively identified main clusters, we calculated pairwise distances between all the stimuli in the MDS plot. We then compared the average distance between clusters to the average distance within clusters using the ratio (as $D=M S_{\text {between }} / M S_{\text {within, where }} M S_{\text {between }}$ is the mean-squared distance, or variance of distances, between the three clusters and $M S_{\text {within }}$ is the average variance of within-cluster distances). The $D$ ratio of 403 for the MDS plot shown in Fig. 9 was highly significant $(P<0.001$, randomization test).

\section{Relative contributions of different patterns of}

correlation: Principal components analysis

We used PCA to assess the relative contributions of different correlation patterns evident in the MDS analysis. The response variation accounted for by the ten most influential principal components is shown in Fig. 10A. The first component accounted for $43 \%$ of the variation in the data, the first two accounted for $69 \%$, and the first eight accounted for $90 \%$ of the response variation. Thus, a small number of response correlation patterns (principal components), each accounted for a much greater proportion of the population response than the $0.78 \%$ that would be expected by chance (given that there were 128 principal components associated with the $128 \times 128$ stimulus input matrix).

Fig. 10B shows the loadings of the stimuli on the first two principal components. The loadings of individual stimuli on a given principal component represent the extent to which the population responses to a given stimulus is correlated with (or, equivalently, is influenced by) the given component. The pattern of correlated and anticorrelated loadings on the two components jointly identify the three stimulus clusters identified by MDS (Fig. 9), in that the first component separates the blue cluster from the remaining stimuli, and the second component segregates the green cluster from the other stimuli (also see legend to Fig. 10B). This was confirmed by ordination of the components (see Sneath \& Sokal, 1973), in which the loadings of the stimuli of the two components were plotted against one another. The clusters of the ordinated plot were similar, and the cluster memberships identical, to the MDS plot shown in Fig. 9 (cophenetic correlation, 0.96; data not shown).

\section{Stimulus similarities underlying response correlations}

Together, the PCA and MDS analyses elucidate some of the similarities among the stimuli that underlie the correlated re- 


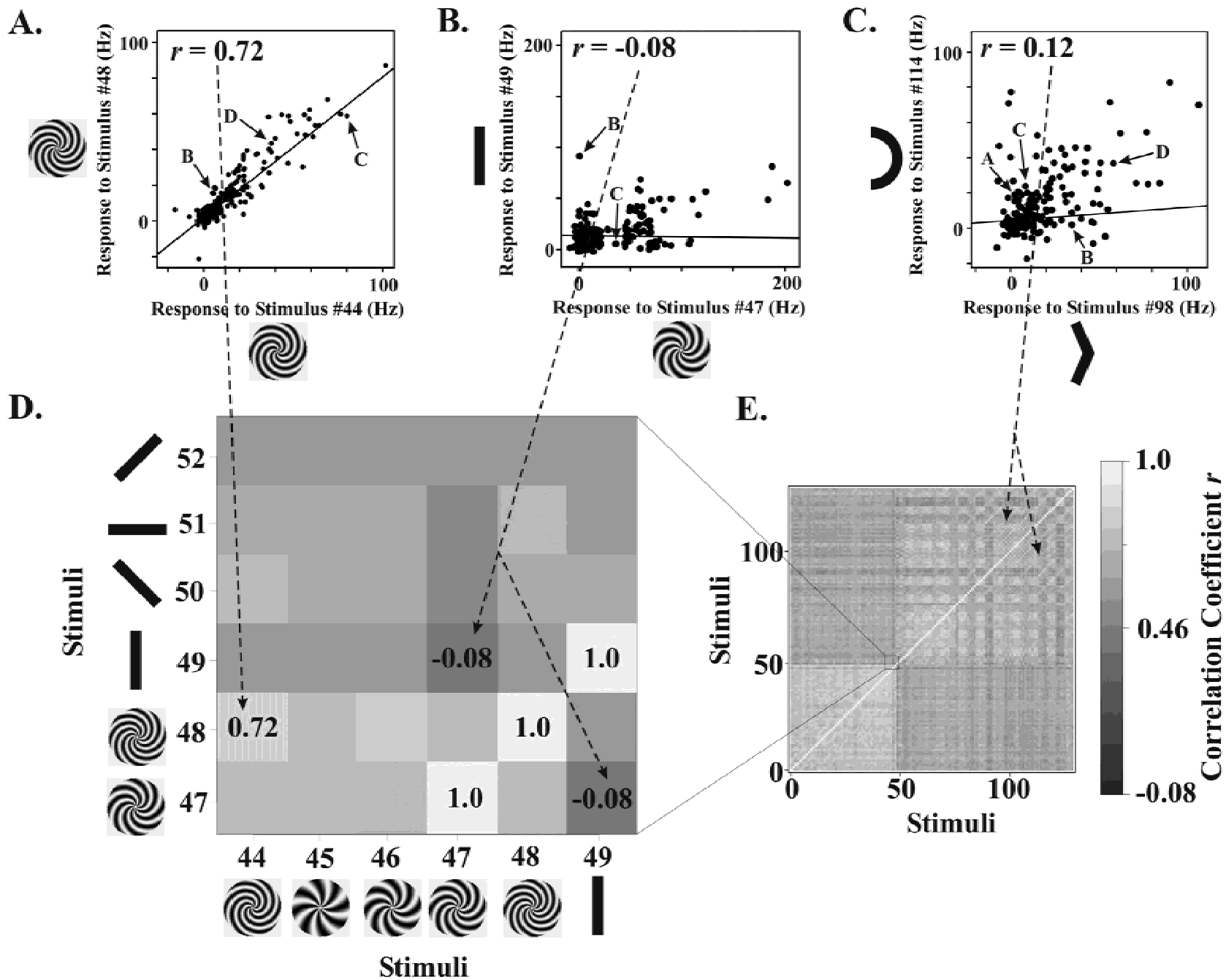

Fig. 8. Constructing the correlation matrix. Panels A-C each show the responses of V2 neurons (dots) to selected pairs of stimuli plotted against each other. The responses of exemplar cells in Fig. 2A-2D are indicated by arrows where possible. The lines represent the best-fitting regression lines; the correlation coefficient $r$ is a measure of deviation of the responses from this line. Panels $\mathrm{E}$ and $\mathrm{D}$ show the entire $128 \times 128$ correlation matrix and a magnified portion thereof, respectively, plotted according to the grayscale on right. In all panels, stimuli are numbered and oriented as in Fig. 2. To construct the correlation matrix, the $r$ value for each possible pair of stimuli was calculated and assigned to the corresponding element of the correlation matrix (dashed arrows). The elements along the diagonal from lower left to upper right represent the correlation of the responses to a given stimulus with themselves $(r=1.0)$. The matrix is symmetrical about this diagonal, since $r(i, j) \equiv r(j, i)$ for any two stimuli $i$ and $j$. Note that $r$ values are low as long as the overall correlation between the responses to the two stimuli is low (panels B and C), even if the responses of subsets of cells are correlated to a greater degree (panel B).

sponses. The response correlations which lead to the clustering of grating stimuli with one another, and apart from the contours, may include contributions from low-level stimulus characteristics like mean luminance, which differed between the two sets of stimuli (see Methods), or from higher order similarities of shape, or from both. However, mean luminance is unlikely to fully account for the response correlations, since many contour stimuli load similarly to the gratings on both the first and the second principal components. Instead, the loading patterns suggest that higher level shape characteristics contribute substantially to the response correlations between gratings and some contours. For instance, many large semicircles, three-quarter arcs, and angles load similarly to the gratings on the first component, suggesting that curvature or orientation differences contribute to this principal component.
The loadings of the contour stimuli are correlated with stimulus size along the second principal component (Fig. 10B, panel 2). Similar results were obtained when MDS or PCA were repeated using only the responses to contour stimuli (see below), confirming that stimulus size contributes substantially to the second principal component. The pronounced differences between the loadings of intersections versus those of most of the large angles and arcs along the second component suggests that the presence or absence of contour junctions is an important factor as well.

Analysis of responses to contours and gratings alone

We analyzed the responses to contour and to grating stimuli separately from each other using MDS and PCA. The MDS of 


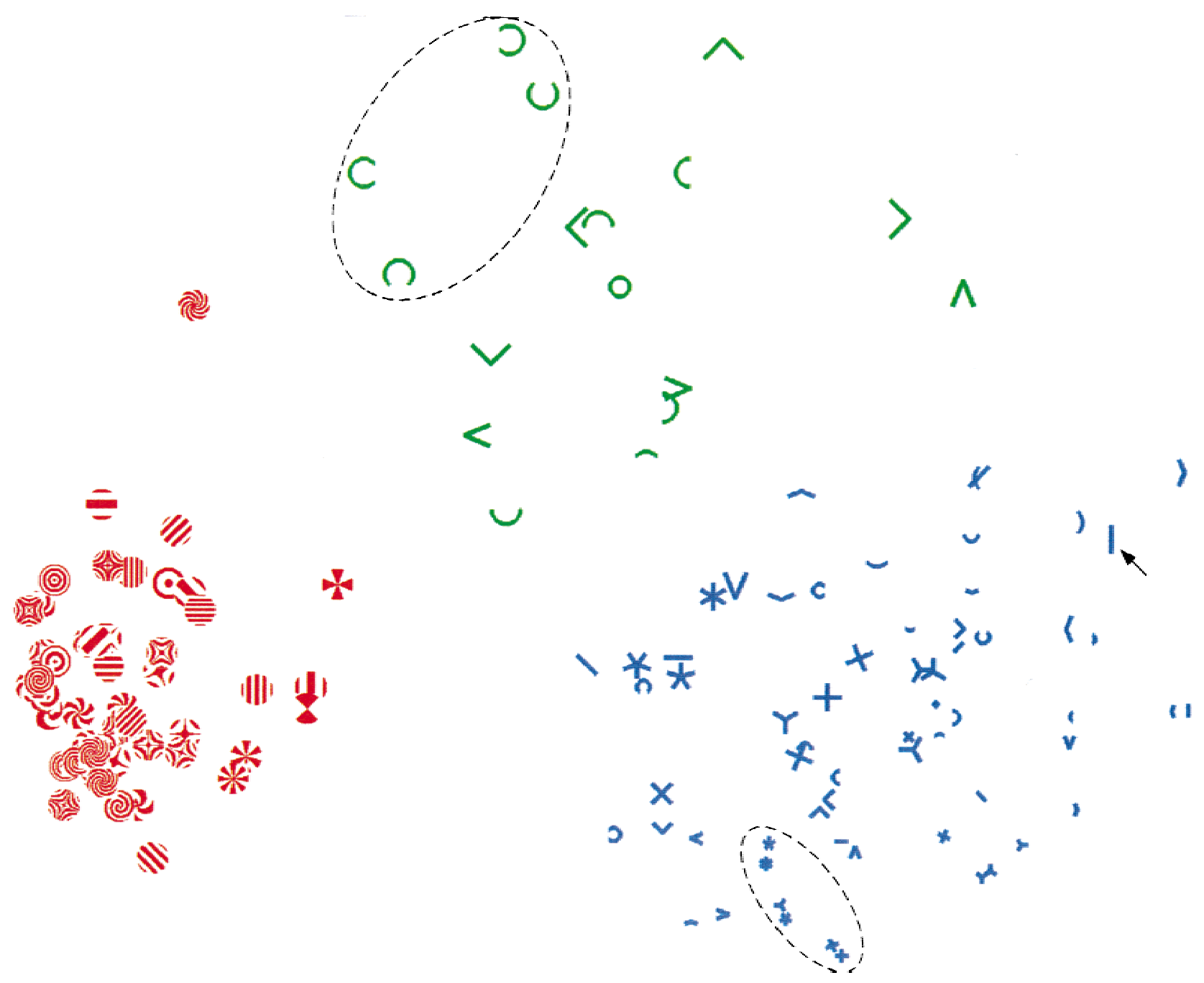

Fig. 9. Metric multidimensional scaling (MDS). Using MDS, the 128 stimuli were plotted so that stimuli which elicited similar responses from the V2 cell population are clustered together and the stimuli which elicit disparate responses are dispersed correspondingly father apart. The two axes represent arbitrary dimensions used by MDS. Three clusters of stimuli, denoted by arbitrary colors, red, green, and blue, were identified based on their spatial separation from each other. The dashed ellipses denote two potential subclusters. The preferred bar is denoted by the arrow.

contour responses produced two clusters which were very similar to the two clusters of contour stimuli (i.e. green and blue clusters) in Fig. 9 (cophenetic correlation $r_{\mathrm{C}}, 0.93$; data not shown). The PCA of the contour responses (Fig. 11) revealed stimulus similarities not readily apparent from the PCA of grating and contour responses combined. Seven principal components exceeded the contribution to response variation expected by chance (arrow and dashed line in Fig. 11A) and together accounted for $88 \%$ of the response variation. Fig. 11B illustrates the loadings of each of these components in composite form, where the stimuli were weighted according to their loading on a given component and overlaid atop each other (see Methods and also Turk \& Pentland, 1991). The first principal component (panel B1), which accounted for more than half of the total response variance, had positive loadings (bright pixels) dominated by bar and angle stimuli and negative loadings (dark pixles) by circular and arc stimuli. Of the top seven principal components, curvature was a predominant shape characteristic in five (panels 1, 2, 5, 6, \& 7), indicating that similarity of curvature was an important determinant of the population response to contour stimuli. Interestingly, panels 5 and 6 were each dominated by arcs of opposite polarity (up vs. down in panel 5; left vs. right in panel 6). Angles and intersections are also important contributors, given their relative prominence in at least two of the top seven components (panels $3 \& 4$ ).

The MDS of grating responses alone resulted in a single cluster similar to the grating cluster (i.e. red cluster) shown in Fig. 9 (cophenetic correlation $r_{\mathrm{C}}, 0.98$ ) and did not reveal any obvious subclustering among the grating stimuli (data not shown). Similarly, PCA of grating responses failed to reveal any pronounced subpatterns of response similarity among the grating stimuli (not shown). Altogether, the response similarity patterns within the grating and contour stimuli were consistent, whether assessed separately or together by MDS or by PCA. This suggests that the patterns were intrinsic to the population responses, and not an artifact of a particular analysis procedure. 


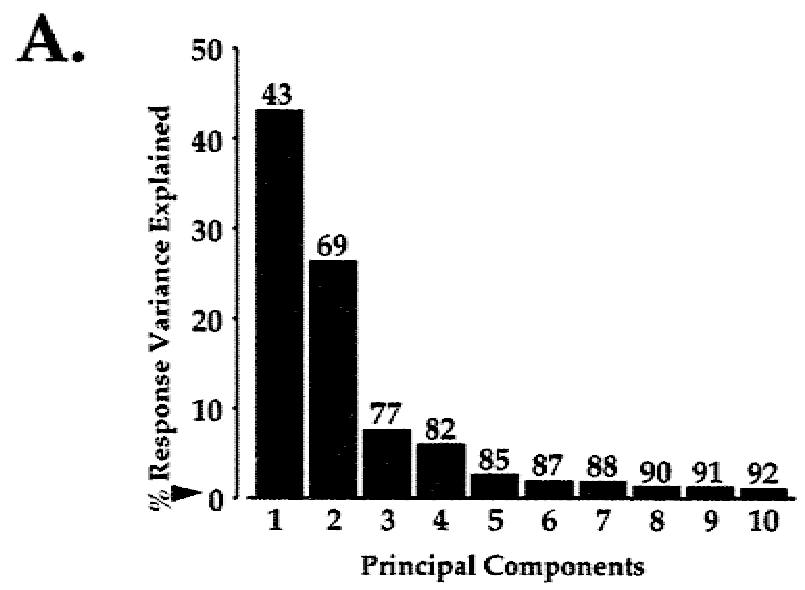

B.

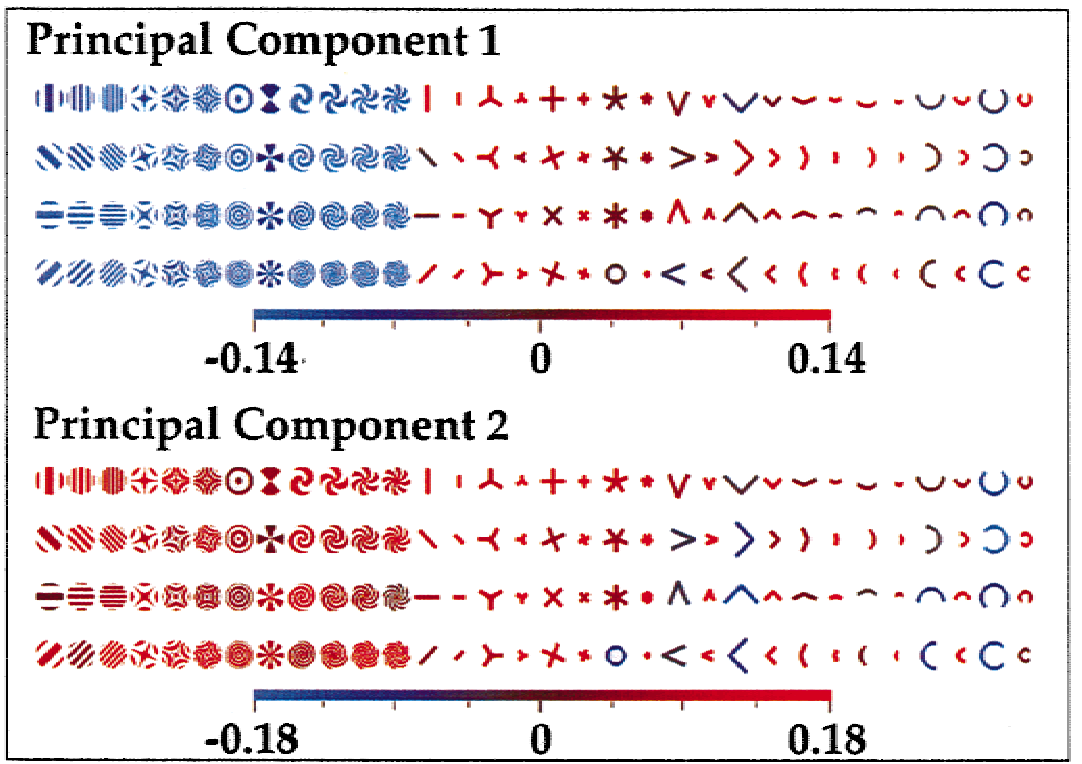

Fig. 10. Principal components analysis (PCA). (A) Proportion of the response variation accounted for by the top principal components. The height of each bar denotes the percentage of the response variation accounted for by the given principal component. The numbers atop the bars denote the cumulative percentages of the response variation accounted for by successive principal components. The arrowhead and the dashed line denote the expected level of response variation accounted for by each principal component if the population response were random. (B) The loadings of individual stimuli on the top two principal components. The color of a given stimulus represents its loading on, or its correlation with, the given principal component according to the corresponding linear color scale at the bottom, where red denotes positive correlation and blue represents anticorrelation. Note that the stimuli positively correlated with the first principal component (panel 1) mostly constitute the blue cluster, and the anticorrelated stimuli belong to the remaining two clusters, in Fig. 9. Note also that the stimuli anticorrelated with the second principal component (panel 2) constitute the green cluster in Fig. 9.

Shape representation among subsets of $V 2$ cells

The specific pattern of response correlations revealed by the MDS analysis might in principle have been dominated by a subpopulation of V2 cells or, alternatively, may reflect characteristics that are widely distributed across the V2 population. To distinguish between these two scenarios, we studied the extent to which randomly chosen subsets of V2 cells exhibited the same response correlation patterns as the overall population.

We randomly sampled the 180 cells at 17 different sample sizes (10-170 cells per sample, in increments of ten cells), generated an MDS plot using each sample, and used cophenetic correlation (see Methods) to asses the similarity between the given MDS plot and the original MDS plot generated using all 180 cells. The average cophenetic correlation $( \pm \mathrm{SD})$ for the $10^{3}$ repetitions at each of the sample sizes is shown in Fig. 12. When only ten cells were used, the resulting MDS plots differed substantially from the original MDS plot generated using all 180 cells (mean $r_{\mathrm{C}}, 0.36$; SD, 0.15). The correlation with the original MDS plot increased rapidly to correlation values of 0.5 for 20 cells (SD 0.14), 0.68 for 45 cells, and 0.86 for 90 cells (SD 0.03). Thus, the response similarities were largely independent of the particular subset of V2 cells analyzed, as long as a moderate number of cells were sampled, indicating that the underlying response similarities were a widespread property of V2 cells. 
A.

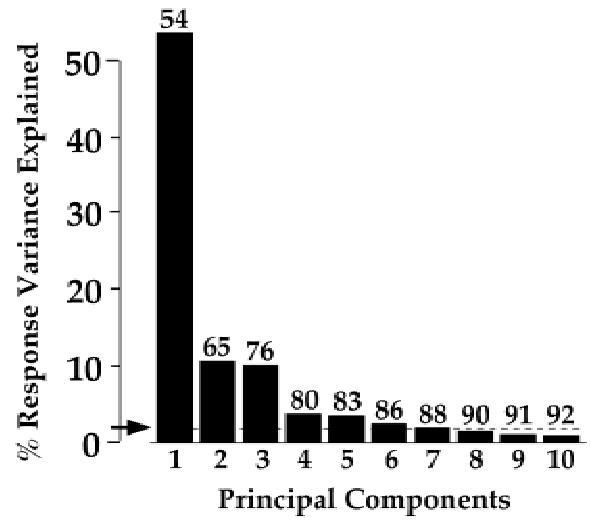

B.
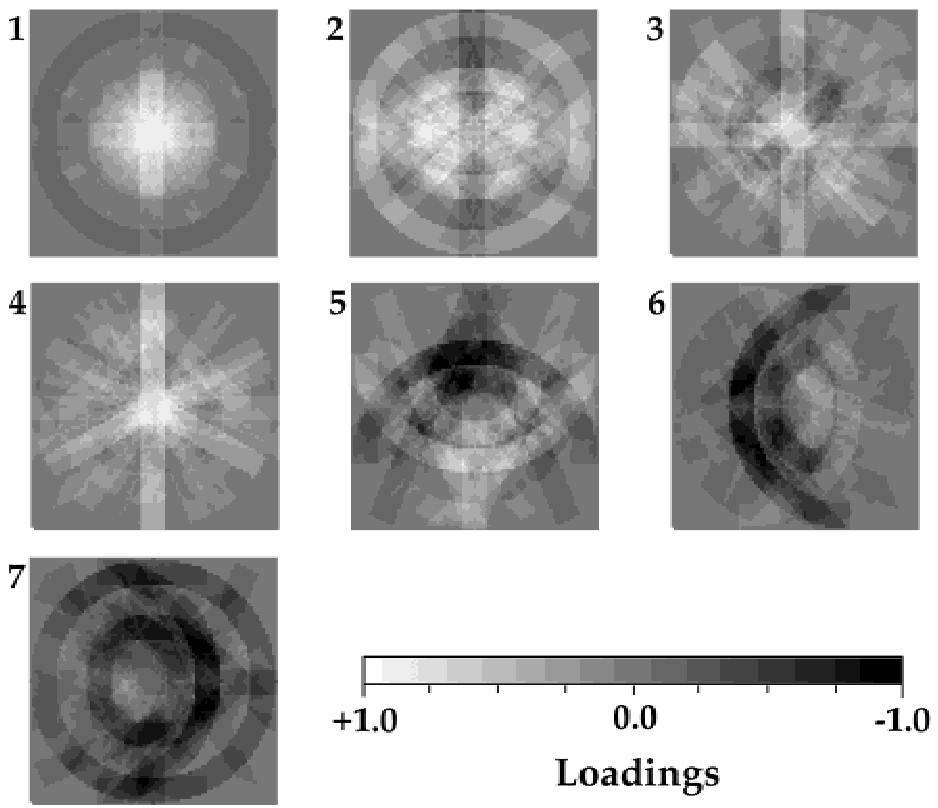

Fig. 11. PCA of the population response to the contour stimuli. (A) Proportion of the response variation accounted for by the top ten principal components. The height of each bar denotes the percentage of the response variation accounted for by the given principal component. The numbers atop the bars denote the cumulative percentages of the response variation accounted for by successive principal components. The arrow and the dashed line both denote the expected level of response variation accounted for by each principal component if the population response were random. (B) The loadings of individual stimuli on the top seven principal components in composite form. The numbers at top left of each composite denotes corresponding principal component. To create a given composite, each contour stimulus was drawn according to its loading on the given component according to the gray scale at bottom using arbitrary stimulus dimensions (preferred bar length and widths of 1.0 and 0.1 receptive-field diameters, respectively). The resulting 80 gray scale images were averaged across all pixels, so that the gray value of a given pixel denotes its weighted average loading on the given component. Neutral gray (background color) represents an average loading of zero, and brighter and darker shades of gray represent positive and negative average loadings, respectively. See Methods for details.

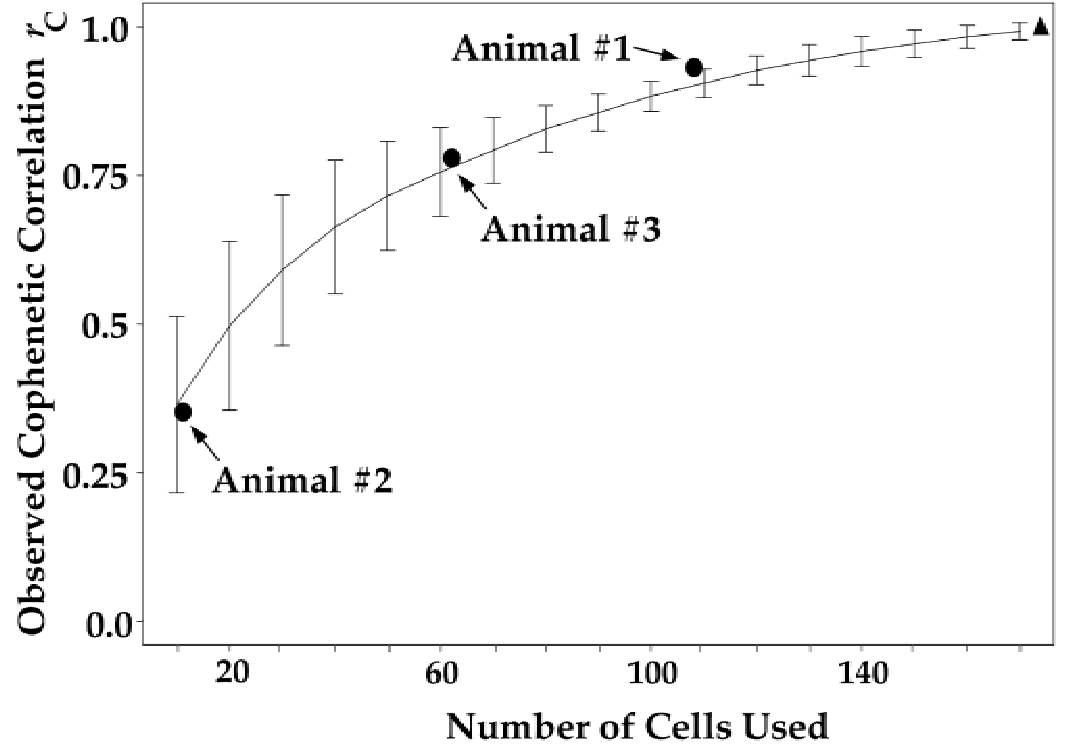

Fig. 12. Shape representation in random subsets of V2 cells. MDS plots were generated using random V2 cell samples of systematically varying sizes. The correlation of each such plot to the original MDS plot in Fig. 8 was measured using cophenetic correlation. The mean cophenetic correlation coefficients $r_{\mathrm{C}}$ (averaged from $10^{3}$ repetitions for each sample size) are plotted here as a function of sample size. Error bars represent standard deviations. The asterisk denotes the average $r_{\mathrm{C}}$ value (also from $10^{3}$ rounds of randomization) that resulted when all 180 cells were used, but the response similarities were randomized among the stimuli. The $r_{\mathrm{C}}$ values calculated from using only the 108 cells from animal \#1, only the 11 cells from animal \#2, or only the 61 cells from animal \#3 are also indicated (dots). The observed $r_{\mathrm{C}}$ value from each animal is indistinguishable from the $r_{\mathrm{C}}$ value expected from the number of cells contributed by that animal (two-tailed $t$-tests, $P>0.05$, not shown). 


\section{Discussion}

Role of V2 cells in shape analysis and image segmentation

Our results indicate that the responses of individual V2 cells convey information about many characteristics of visual contours, including geometric shape (curvature, intersections, and angles) as well as size and orientation. Many individual V2 cells also convey some information about textural characteristics, insofar as they are selective for non-Cartesian or sinusoidal gratings. These observations are supported by recent findings that many V2 neurons have spatially segregated subregions with different preferred orientations (Anzai \& Van Essen, 2001, 2002).

In the present study, the population varied widely in terms of selectivity for various shape characteristics. Also, the population as a whole shows only modest biases in terms of responsiveness to particular subsets of shapes. This suggests that V2 cells sample the space of grating and contour stimuli widely and are not specialized for analyzing a narrow subset of shape characteristics of contours and/or textures within our stimulus set. This apparent diversity in analysis strategies may reflect the diversity of perceptually relevant shape cues in natural images (Cho et al., 2000; Geisler et al., 2001; Sigman et al., 2001; also see Field, 1987; Simoncelli \& Olshausen, 2001).

Besides its involvement with monocular shape cues, V2 is engaged in the analysis of several other low-level dimensions, including stereoscopic disparity, color, and motion (see Van Essen \& Gallant, 1994; and Roe \& Ts'o, 1997 for a review). That V2 should be engaged in such a broad spectrum of physiological analyses is consistent with its hierarchical position as the primary recipient of inputs from $\mathrm{V} 1$ and the source of projections to numerous visual areas in both the ventral and dorsal processing streams (Desimone \& Ungerleider, 1987; Felleman \& Van Essen, 1991). It also fits with computational arguments that early visual processing involves multiple states of image analysis to generate generic shape representations (Marr, 1982; Yuille \& Ullman, 1995).

\section{Significance of the response correlations and} low-dimensional representations

The fact that a modest number of principal components account for most of the response variation in the population response indicates that V2 cells can represent most of the shape information in our particular stimulus set by a smaller number of basis functions than would be required if the population responses were highly decorrelated. This supports the hypothesis of a low-dimensional representation as plausible general strategy for shape representation in V2. This strategy can provide a simplified, or low-dimensional, representation of the visual input while preferentially retaining perceptually relevant information, analogous to the manner in which stenography represents words using a smaller number of symbols with little ambiguity. Computational studies suggest that low-dimensional representation, that is, simplification of the representation to retain salient information and discard less salient information, is an effective strategy of processing input data of high complexity using limited data processing resources (Seung \& Lee, 2000; also see Roweis \& Saul, 2000; Tennenbaum et al., 2000). This strategy also facilitates generalization across varying inputs (Oja, 1995; Seung \& Lee, 2000). In our context, this would signify that the V2 cell population can tolerate small geometric variations in contour and texture cues while extracting relevant higher order shape information.
Previous studies have suggested that neuronal populations in macaque inferotemporal cortex encode faces along a small number of psychophysically salient dimensions (Young \& Yamane, 1992). There is also evidence that major aspects of population responses in other parts of the brain can be captured in a small number of response dimensions related to the angular eye position or head direction (McFarland \& Fuchs, 1992; Taube, 1998). Our results suggest that an analogous low-dimensional population representation of shape characteristics may be used in early visual cortical processing stages.

It is naturally of interest to ascertain whether the dimensionality with which complex shapes are represented varies markedly at different levels of the visual hierarchy. Insights regarding this issue should be attainable by systematically analyzing multiple visual areas using a large but stereotyped family of shapes. At the level of retinal ganglion cells and lateral geniculate nucleus (LGN) neurons, it seems likely that the dimensionality would be lower than what we encountered in V2, because neurons with stereotyped center-surround receptive fields should respond similarly to many different shapes, and a high fraction of response variance would be captured by a few principal components. Whether there are changes in dimensionality at different levels of the cortical hierarchy will be more challenging to determine, for technical reasons that include the large interareal differences in average receptive-field size and the difficulty of selecting stimulus sets that are effective in spanning a large fraction of the relevant stimulus dimensions in each area.

Another intriguing issue is whether the dimensionality of the sensory representation changes significantly over the time course of neural responses. For example, in the vertebrate olfactory system, the mitral cell population response is initially lowdimensional, but becomes decorrelated and higher in dimensionality during the responses to sustained odorant stimuli (Friedrich \& Laurent, 2001). This may allow the generation of an initial coarse-grained representation, useful for rapid stimulus classification, followed by a more slowly developing finer-grained representation useful for finer-grained discrimination tasks. In visual cortex, time-dependent changes in the selectivity of neuronal responses occur in V1 and V2, suggesting that increases in time-dependent dimensionality may occur for visual representations as well (Menz \& Freeman, 2003; Hegdé \& Van Essen, unpublished data)

\section{Acknowledgments}

We thank Michael Ty for help with neurophysiological recording, and Drs. Charles Anderson, James Cheverud, and Joseph O'Sullivan for useful discussions. We thank Gregory DeAngelis, Jack Gallant, Rachel Kalmar, Jonathan Nassi, and Gene Stoner for helpful comments on the manuscript. This work was supported by NIH grant EY-02091 to D.C. Van Essen.

\section{References}

AnZai, A. \& Van Essen, D.C. (2001). Receptive field substructure of monkey V2 neurons in the orientation domain. Society for Neuroscience Abstracts Vol. 27, \#286.5. Online (wwww.sfn.org)

AnZaI, A. \& VAN Essen, D.C. (2002). Receptive field structure of orientation selective cells in monkey V2. Society for Neuroscience Abstracts. \#720.12. Online (www.sfn.org)

BERgEn, R. (1991). Theories of visual texture perception. In Vision and Visual Dysfunction, Vol. 10B, ed. Regan, D., pp. 114-134. New York: Macmillan.

Brase, C.H. \& Brase, C.P. (1995). Understandable Statistics. Lexington, Massachusetts: DC Heath and Co. 
Cho, R.Y., YAng, V. \& Hallett, P.E. (2000). Reliability and dimensionality of judgments of visual textured materials. Perceptual Psychophysics 62, 735-752.

Cox, T.F. \& Cox, M.A. (1994). Multidimensional Scaling. New York: Chapman and Hall.

Desimone, R. \& UnGerleider, L.G. (1987). Neural mechanisms of visual processing in monkeys. In Handbook of Neuropsychology, Vol. 2, ed. Boller, F. \& Grafman, J., pp. 267-299. Amsterdam: Elsevier

Dunteman, G.H. (1989). Principal Components Analysis. Newbury Park, California: Sage Publications.

EAgelson, R. (1992). Measurement of the 2D affine Lie group parameters for visual motion analysis. Spatial Vision 6, 183-198.

Felleman, D.J. \& VAN Essen, D.C. (1991). Distributed hierarchical processing in the primate cerebral cortex. Cerebral Cortex 1, 1-47.

FIELD, D.J. (1987). Relations between the statistics of natural images and the response properties of cortical cells. Journal of the Optical Society of America A 4, 2379-2394.

FIELD, D.J. (1995). Visual coding, redundancy, and and "feature detection". In Handbook of Brain Theory and Neural Networks, ed. ArвIB, M.A., pp. 1012-1016. Cambridge, Massachusetts: MIT Press.

Friedrich, R.W. \& Laurent, G. (2001). Dynamic optimization of odor representation by slow temporal patterning of mitral cell activity. Science 291, 889-894.

Gallant, J.L., Braun, J. \& Van Essen, D.C. (1993). Selectivity for polar, hyperbolic, and Cartesian gratings in macaque visual cortex. Science 259, 100-103.

Gallant, J.L., Connor, C.E., Rakshit, S., Lewis, J.W. \& Van Essen, D.C. (1996). Neural responses to polar, hyperbolic, and Cartesian gratings in area V4 of the macaque monkey. Journal of Neurophysiology 76, 2718-2739.

Geisler, W.S. \& Super, B.J. (2000). Perceptual organization of twodimensional patterns. Psychological Review 107, 677-708.

Geisler, W.S., Perry, J.S., Super, B.J. \& Galogly, D.P. (2001). Edge co-occurrence in natural images predicts contour grouping performance. Vision Research 41, 711-724.

Grossberg, S. (1987). Cortical dynamics of three-dimensional form, color, and brightness perception. Perceptual Psychophysics 41, 87-158.

Hegdé, J. \& VAN Essen, D.C. (2000). Selectivity for complex shapes in primate visual area V2. Journal of Neuroscience 20, RC61-66.

HuberTy, C.J. \& Morris, J.D. (1989). Multivariate analysis versus multiple univariate analyses. Psychological Bulletin 114, 145-161.

Kachigan, S.K. (1991). Multivariate Statistical Analysis. New York: Radius Press.

Kruskal, J.B. \& Wish, M. (1978). Multidimensional Scaling. Newbury Park, California: Sage Publications.

Manly, B.F.J. (1991). Randomization and Monte Carlo Methods in Biology. New York: Chapman and Hall.

Marr, D. (1982). Vision. New York: W.H. Freeman and Co.

McFarland, J.L. \& Fuchs, A.F. (1992). Discharge patterns in nucleus prepositus hypoglossi and adjacent medial vestibular nucleus during horizontal eye movement in behaving macaques. Journal of Neurophysiology 68, 319-332.

Menz, M.D. \& Freeman, R.D. (2003). Stereoscopic depth processing in the visual cortex: A coarse-to-fine mechanism. Nature Neuroscience 6(1), 59-65.

OJA, E. (1995). Principal component analysis. In Handbook of Brain Theory and Neural Networks, ed. ArbiB, M.A., pp. 753-756. Cambridge, Massachusetts: MIT Press.
Osherson, D.N., Kosslyn, S.M. \& Hollerbach, J.M. (ed.) (1995). Visual Cognition and Action. Cambridge, Massachusetts: MIT Press.

Pasupathy, A. \& Connor, C.E. (1999). Responses to contour features in macaque area V4. Journal of Neurophysiology 82, 2490-2502.

Perona, P. (1991). Deformable kernels in early vision. IEEE Conference on Vision Pattern Recognition 761, 222-227.

RoE, A.W. \& Ts'o, D.Y. (1997). The functional architecture of area V2 in the macaque monkey. Physiology, topography and connectivity. In Cerebral Cortex, Vol. 12. Extrastriate Cortex in Primates, eds. RockLAND, K.S., KaAs, J.H. \& Peters, A., pp. 295-333. Plenum.

Rogers-Ramachandran, D.C. \& Ramachandran, V.S. (1997). Psychophysical evidence for boundary and surface system in human vision. Vision Research 38, 71-77.

Rolls, E.T. \& ToveE, M.J. (1995). Sparseness of the neuronal representation of stimuli in the primate temporal cortex. Journal of Neurophysiology 73, 713-726.

RoweIs, S.T. \& SAUL, L.K. (2000). Nonlinear dimensionality reduction by locally linear embedding. Science 290, 2323-2326.

Savitz, D.A. \& Olshan, A.F. (1995). Multiple comparisons and related issues in the interpretation of epidemiological data. American Journal of Epidemiology 142, 904-908.

SEUnG, H.S. \& LEE, D.D. (2000). The manifold ways of perception. Science 290, 2268-2269.

Sigman, M., Cecchi, G.A., Gilbert, C.D. \& Magnasco, M.O. (2001). On a common circle: Natural scenes and Gestalt rules. Proceedings of the National Academy of Sciences of the U.S.A. 98, 1935-1940.

Simoncelli, E.P. \& Olshausen, B.A. (2001). Natural image statistics and neural representation. Annual Review of Neuroscience 24, 1193-1215.

Sneath, P.H.A. \& Sokal, R.R. (1973). Numerical Taxonomy. San Francisco, California: W.H Freeman and Co.

Snedecor, G.W. (1934). Analysis of Variance and Covariance. Ames, Iowa: Ames.

SoKal, R.R. \& RohlF, F.J. (1962). The comparison of dendrograms by objective methods. Taxon 11, 33-40.

TAuBE, J.S. (1998). Head direction cells and the neurophysiological basis for a sense of direction. Progress in Neurobiology 55, 225-256.

Tennenbaum, J.B., DeSilva, V. \& Langford, J.C. (2000). A global geometric framework for nonlinear dimensionality reduction. Science 290, 2319-2323.

Thompson, J.R. (1998). Invited commentary: Re: "Multiple comparisons and related issues in the interpretation of epidemiological data". American Journal of Epidemiology 147, 801-806.

Turk, M. \& Pentland, A. (1991). Eigenfaces for recognition. Journal of Cognitive Neuroscience 3, 71-86.

Van Essen, D.C. \& Gallant, J.L. (1994). Neural mechanisms of form and motion processing in the primate visual system. Neuron 13, 1-10.

VinJe, W.E. \& Gallant, J.L. (2000). Sparse coding and decorrelation in primary visual cortex during natural vision. Science 287, 1273-1276.

Wandell, B.A. (1995). Foundations of Vision. Sunderland, Massachusetts: Sinauer Associates, Inc., pp. 229-238.

Wilkinson, F., Willon, H.R. \& HaBAK, C. (1998). Detection and recognition of radial frequency patterns. Vision Research 38, 3555-3568.

Young, M.P. \& Yamane, S. (1992). Sparse population coding of faces in the infereotemporal cortex. Science 256, 1327-1331.

Yuille, A.L. \& Ullman, S. (1995). Computational theories of low-level vision. In Visual Cognition and Action, ed. Osherson, D.N., Kosslyn, S.M., \& Hollerbach, J.M., pp. 5-40. Cambridge, Massachusetts: MIT Press. 